\title{
The shape of the vibrotactile loudness function: The effect of stimulus repetition and skin-contactor coupling
}

\author{
AMY A. COLLINS and ROGER W. CHOLEWIAK \\ Princeton University, Princeton, New Jersey
}

\begin{abstract}
In the psychophysical literature describing the relationships between physical and psychological magnitudes, as physical intensity increases, perceived intensity often grows much faster near threshold than at higher levels. In this laboratory, however, the loudness curve for sinusoidal vibrotactile stimuli was best fit by a single-limbed function rather than by the expected two-limbed function. In the present study, we measured the growth of vibrotactile loudness of $250-\mathrm{Hz}$ sinusoidal stimuli by the method of absolute magnitude estimation to explore the source of the one- versus two-limbed discrepancy. The number of times that the stimulus was presented was varied, as well as whether the stimulator contacted the skin with constant force or constant penetration. Neither of these manipulations affected the shape of the loudness function consistently. Number of repetitions influenced the shapes of the magnitude estimation functions, but only for a few individuals. Skin-contactor coupling did not affect the shapes of the functions, although the absolute level (vibrotactile loudness) was consistently greater for constant indentation.
\end{abstract}

The exponent of the loudness function is normally reported to be about 0.9 for vibration delivered to the palm of the hand (e.g., Gescheider, 1976; Verrillo, 1974, 1982; Verrillo \& Capraro, 1975; Verrillo \& Chamberlain, 1972; Verrillo, Fraioli, \& Smith, 1969). ${ }^{1}$ However, this represents only the rate of growth for higher stimulus intensities. It has been generally accepted that the form of the function for sinusoidal loudness on the palm changes slope with increasing intensity in the range of $15-25 \mathrm{~dB} \mathrm{SL}$ (e.g., Marks, 1979; Verrillo et al., 1969). Specifically, the rate of loudness growth for weak stimulus intensities is about twice that for the stronger signal levels. Recently, however, several studies done in the Princeton laboratories have examined vibrotactile loudness growth over a wide intensitive range, including near-threshold stimuli, and did not find steep lower limbs in the resulting functions (Collins \& Cholewiak, 1989a, 1989b; Laverty, 1981). The data from these studies were best fit over the full range of stimuli by single-limbed functions. Furthermore, the slopes of these functions were consistent with those reported for only the upper limbs of comparable data in the literature.

Whether the psychophysical function possesses one or two limbs is not an inconsequential issue. The potential differences in apparent magnitude at low intensities can

This study was supported by NIH Grant DC-00076 to Princeton University. Requests for reprints may be sent to the authors at the Department of Psychology, Green Hall, Princeton University, Princeton, NJ 08544-1010. The authors would like to acknowledge the helpful comments made by Larry Marks, Bill Rabinowitz, and an anonymous reviewer. be considerable if the loudness of a low-intensity stimulus predicted by the lower of a two-limbed function is compared with that predicted solely by extrapolation of the upper limb into the low-intensity region. For example, a typical loudness function for a vibrotactile stimulus is plotted in Figure 1, with the solid lines representing the best-fit, two-limbed curve. The figure shows magnitude judgments of $250-\mathrm{Hz}$ vibration on the palm of the hand (data points were taken from Verrillo, 1974, Figure 3). The dashed lines in the graph illustrate the effect of simply extrapolating the high-intensity function down into the lower intensity range for this set of data; the separation between the arrowheads shows the difference in loudnesses for the lowest intensity stimulus (approximately $9 \mathrm{~dB} \mathrm{SL}$ ). In this particular case, the loudness difference predicted by the extrapolation versus that actually obtained for the weakest vibrotactile stimulus is greater than 10:1.

A comparison between the recent studies from this laboratory and those in the literature to shed light on the possible reasons for this discrepancy in the shape of the loudness function revealed two methodological differences. One was the number of times that the test stimulus was presented within a trial. For example, Collins and Cholewiak $(1989 a, 1989 b)$ used a method in which observers based their judgments on only a single presentation of the stimulus. Other researchers, on the other hand, often repeat the stimulus until the observer makes a response (Verrillo, 1982; R. T. Verrillo \& S. J. Bolanowski, personal communication, May 24, 1989). Another difference among studies is the manner in which the skin-contactor relationship (coupling) is maintained. In some studies, the stimulator is mounted on a balance arm in order to maintain a constant force against the skin, usually about $20 \mathrm{~g}$ 


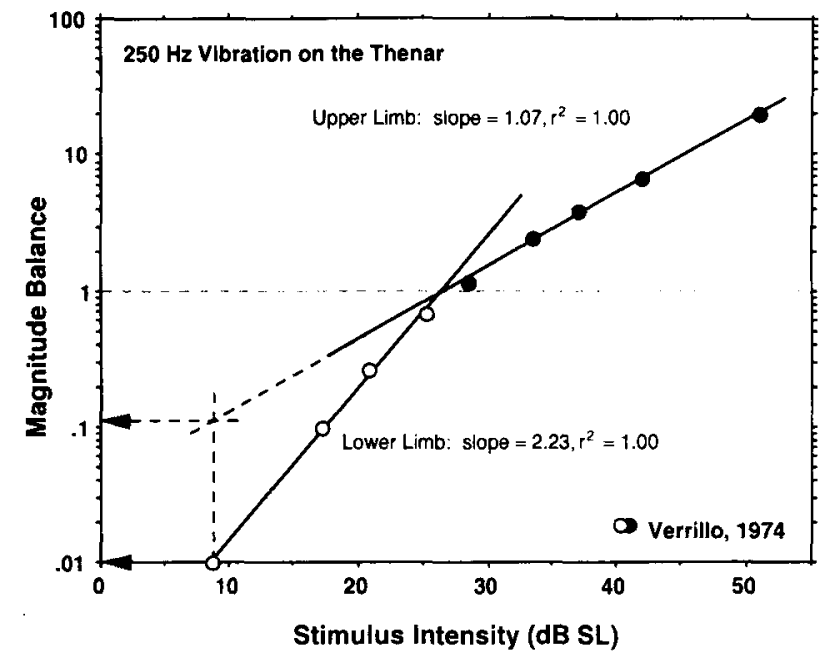

Figure 1. Magnitude balance function for the growth of loudness of $900-$ msec bursts of $250-\mathrm{Hz}$ vibration on the thenar as a function of the intensity of the vibration in decibels sensation level (dB SL). Magnitude balance is a procedure employing both magnitude estimation and magnitude production of stimuli. The contactor area was $0.28 \mathrm{~cm}$ square, and a surround was present. The slope of the power function fitted to the upper portion of the curve is 1.07; that for the lower portion is 2.23. Data points were taken from Verrillo (1974, Figure 3). See Note 1 regarding calculation of slopes.

(e.g., Collins \& Cholewiak, 1989a, 1989b; Craig \& Sherrick, 1969; S. S. Stevens, 1968). In this case, the degree to which the contactor is indented into the skin depends on the size of the contactor, the stiffness of the skin, and the presence of a static surround, as well as other similar factors. Other laboratories (e.g., Bernstein, Schechter, \& Goldstein, 1986; Gescheider \& Wright, 1968; Verrillo, 1962; Verrillo \& Chamberlain, 1972) allow the static force of the contactor to vary while holding constant the amount of indentation into the skin. ${ }^{2}$

The present experiments were designed to determine whether these procedural factors influence the shapes of loudness functions. The loudness of $250-\mathrm{Hz}$ sinusoidal stimuli presented to the palm of the hand was measured by the method of absolute magnitude estimation. In the present study there were two experiments: one in which force was held constant, and one in which the amount of indentation of the contactor into the skin was held constant. All other parameters were identical between the two. In both experiments, there were three conditions varying in the number of times the stimulus was presented to the observer within a trial before a judgment was required.

\section{METHOD}

\section{Apparatus}

The stimuli were bursts of $250-\mathrm{Hz}$ sinusoidal vibration presented to the thenar eminence of the palm of the right hand (the fleshy pad at the base of the thumb). The sinusoidal signal was generated by an Exact 627 function generator and sent to a computer-gated Grason Stadler 829D electronic switch. The resulting burst had a rise-fall time of $25 \mathrm{msec}$ and a duration of $500 \mathrm{msec}$. The signal was passed through a cascaded pair of attenuators, then to a Brüel \& Kjaer 2706 power amplifier, which also matched signal impedance to the Brüel \& Kjaer 4810 Mini Shaker. A PCB 303A accelerometer mounted directly onto the moving element of the vibrator served as the contactor for the skin as well as the monitoring system for the sinusoidal signal. The output of the calibrated accelerometer was passed to a PCB 480D06 power unit/pre-amp, monitored with an oscilloscope, and measured with a Hewlett-Packard 34740A digital voltmeter. Displacement in microns was calculated from the acceleration voltage, and then converted to decibels with reference to 1 micron peak. The contactor was $7 \mathrm{~mm}$ in diameter (area = $0.385 \mathrm{~cm}^{2}$ ) and touched the skin through a 9-mm hole in the center of a rigid plastic plate. The contactor system was maintained at normal skin temperature. In addition, the observers wore headphones that delivered white noise at a comfortable level to mask any ambient sounds.

In the first experiment, the Brüel \& Kjaer 4810 Mini Shaker was mounted onto a beam balance to provide $20 \mathrm{~g}$ of constant force to the thenar eminence of the palm. For the second experiment, the shaker was rigidly mounted so as to protrude through the static surround by approximately $0.5 \mathrm{~mm}$. In both cases, the hand was placed on the contactor and held motionless with a sandbag. The manner in which the constant-indentation condition was established was somewhat different from the method used by other laboratories (see, e.g., Verrillo, 1962). Specifically, others raise the contactor from below the support table until it comes into direct contact with the skin, then continue to raise it until it moves 0.5 or $1.0 \mathrm{~mm}$ farther. This method compensates for differences in skin compliance (or stiffness) that might exist among observers. In our case, the observer placed the thenar eminence on the contactor with sufficient force so that the rest of the pad touched the surround. Here we are assuming some average amount of compliance, but small differences might exist in the equivalent indentation across our observers, which should have only a minor effect on the absolute threshold level (Verrillo, 1966). ${ }^{3}$

\section{Subjects}

The 12 paid observers in each part of the study were Princeton University undergraduate and graduate students. In the first experiment, there were 5 males and 7 females. Of the 12 observers, 10 were naive to magnitude estimation procedures, and 2 had previously participated in another absolute magnitude estimation study in this laboratory. In the second experiment, there were 7 males and 5 females. All but 1 of these observers were naive to magnitude estimation procedures; this subject participated in both experiments. Different observers were used to minimize any effects of familiarity with the stimulus range, and so forth (Poulton, 1979).

\section{Procedure}

In both of the experiments reported here, a brief preliminary training series was conducted to familiarize the observers with the method of absolute magnitude estimation (AME). This series required the observers to make judgments of the magnitude of line lengths, a relatively easy task that appears to stabilize loudness scaling (S. S. Stevens, 1975; Zwislocki, 1983; Zwislocki \& Goodman, 1980). The observers were given AME instructions modeled after those in Zwislocki (1983). Lines were presented by exposing more or less of a 1-m length of 1-mm-thick brown wire at a distance of about $0.7 \mathrm{~m}$ from the observer. The lines ranged in length from $1 \mathrm{~cm}$ to $1 \mathrm{~m}$. The actual lengths presented and their order of presentation were randomly selected.

Each experiment was completed in a single session. After making line-length judgments, vibrotactile threshold was obtained by the method of adjustment in which a single-turn $40-\mathrm{dB}$ attenuator was used to raise or lower the intensity of the signal. The test stimulus was repeated once every $2 \mathrm{sec}$. The observer judged four se- 
ries: two ascending and two descending, presented in a DAAD order. Subsequently, there were three blocks of AME trials that differed from one another only in the number of times in a trial that the stimulus was presented to the observer. In one, the stimulus was presented only once; in another, the stimulus was presented three times. In the third, the stimulus was presented as many times as the observer wished. The order of the conditions was randomly selected and counterbalanced across observers. When the stimulus was presented more than once, there was a delay of $1,500 \mathrm{msec}$ between the 500 -msec bursts of vibration. Before each block, the observer was given AME instructions and was told how many presentations of the stimulus to expect. Within a block, each intensity level (increasing from threshold in 5-dB steps with a possible range of from 5 to $50 \mathrm{~dB} \mathrm{SL}$ ) was presented twice in random order. In addition, four of these levels were randomly chosen to be also presented as practice at the very beginning of the block. Because of equipment limitations that restricted the highest intensity levels possible, the observers with relatively high thresholds had a proportionally limited stimulus intensity range (the range of 5-40 dB SL was used in at least two out of three blocks for 3 observers in the first experiment and 5 observers in the second).

Two criteria were selected to identify curves that were best fit by two-limbed functions, allowing for an objective test of the shapes of the loudness functions. One was based on a characteristic that appears to be true of two-limbed functions found in the literature: the slope of the lower limb is about twice as large as that for the upper limb. For our calculation, a ratio greater than 1.8 was considered necessary to define a 2-to-1 difference in slope between the lower and upper limbs. The second was that two limbs fit the data significantly better than would a single-limbed function. This statistical criterion was examined by analyzing the lower and upper ranges of data points with a set of tests that fit regression lines to the data and assessed the differences between them (Wiggans, Andrews, \& Sahgal, 1983; Woodward, 1972). ${ }^{4}$

\section{RESULTS}

Thresholds obtained in the two experiments were quite similar, despite the differences in the contact conditions and observer populations. In the constant-force group, the mean threshold was $-14.9 \mathrm{~dB}$ re 1 micron peak $(S E=$ $1.7 \mathrm{~dB}$ ) whereas threshold for the constant-indentation group averaged $-13.6 \mathrm{~dB}$ re 1 micron peak $(S E=$ $1.5 \mathrm{~dB}$ ). These are equivalent to 0.22 and 0.25 micron peak, respectively. Equivalent threshold functions for these coupling arrangements have been found in previous studies using the same parameters (Cholewiak, Sherrick, \& Collins, 1992; Lamoré \& Keemink, 1988; see also Note 2). The threshold values are somewhat lower than those reported by other laboratories for the same parameters (averaging about $-6.0 \mathrm{~dB}$ for Verrillo, 1974; Verrillo \& Capraro, 1975; Verrillo \& Chamberlain, 1972, with the coupling arrangement of constant indentation).

When the observers were allowed to control the number of times that the stimulus was presented to them (the ad-lib condition), the average number of presentations was approximately three (constant force $=3.12$; constant indentation $=2.93$ ), about the same as the largest number of repetitions allowed in the restricted conditions. It is notable that Verrillo (1982) reported that in a similar adlib condition, observers typically allowed themselves three presentations before making a judgment and going on to the next stimulus.

The group data for both experiments can be seen in Figure 2 , in which the geometric means of the absolute magnitude estimates are plotted as a function of signal intensity in decibels re threshold. Examining the data by eye, no compelling evidence of a bend suggesting a two-limbed function appears in any of the conditions. Indeed, these group data failed to meet both criteria outlined in the procedures. ${ }^{5}$ Thus, the best-fit curves were calculated by the method of least squares for the single-limbed functions, as shown. For comparative purposes, the geometric means of the magnitude estimates of sinusoidal stimuli also best fit by single-limbed functions from two similar studies done in the Princeton laboratories are plotted in the figure (from Collins \& Cholewiak, 1989a, and Laverty, 1981). Laverty measured the growth of loudness of 250 $\mathrm{Hz}, 20$-msec sine bursts presented to the thenar eminence with an 8-mm-diam contactor (without a surround) resting on the skin with a force of $20 \mathrm{~g}$. The data reported by Collins and Cholewiak (1989a) were obtained with the same apparatus and stimulus conditions that were used in the present study. The data from both of these past studies are remarkably similar to those obtained in the present one. The slopes (exponents) for these two functions were identical: 0.84 , and within the expected range for upper-limb exponents for $250-\mathrm{Hz}$ vibrotactile stimuli on the palm.

Although the skin-contactor coupling arrangements yielded equivalent threshold data, in Figure 2 there appears to be a small loudness difference between the filled and open symbols. When the functions were compared statistically, no differences in the slopes of the functions were found, but the loudness differences between the coupling arrangements were significant [one presentation, $F(1,220)=40.85, p \leq .05$; three presentations, $F(1,223)=20.19, p \leq .05 ;$ ad lib, $F(1,221)=17.02$, $p \leq .05$ ]. It is possible that the differences in absolute loudness levels are artifacts of different number usage by the two groups of observers and are not meaningful. Alternatively, the difference in loudness may result from the difference in static force that exists between the two contactor conditions. In the first experiment, the contactor rested against the skin with a static force of $20 \mathrm{~g}$. In the second experiment, the contactor produced a $0.5-\mathrm{mm}$ constant indentation into the skin of the thenar while static force was not controlled. In order to maintain that indentation, we have determined that approximately $35 \mathrm{~g}$ of static force is necessary. Consequently, the net forces involved were greater in the second experiment by approximately $15 \mathrm{~g}$. Examining the data from Green and Craig (1974) and Craig and Sherrick (1969), it appears that this difference in static force alone could have produced an increase in loudness of about $6 \mathrm{~dB}$ in favor of the staticindentation group. Indeed, the differences seen here are on the order of $6 \mathrm{~dB}$. It should be noted that changes in static force can affect sensation magnitude, yet not influ- 


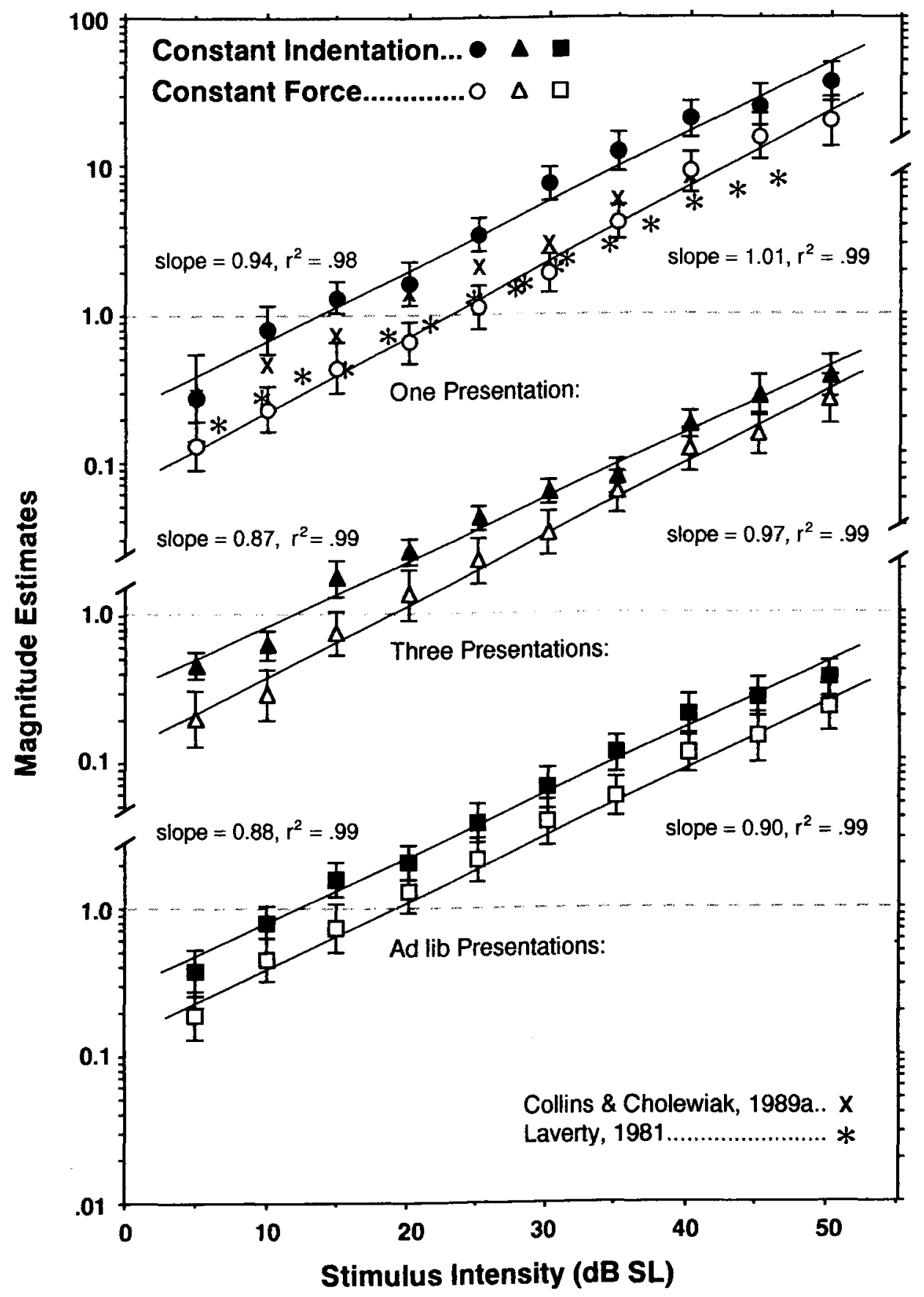

Figure 2. The geometric means of the absolute magnitude estimates from both experiments for 250Hz sinusoidal bursts, plotted here as a function of signal intensity in decibels sensation level (dB SL). The observers made their judgments after one, three, or ad-lib presentations of the stimulus. Note that the functions are offset vertically from one another for clarity by $2 \log$ units, and that the data are best nit by single-limbed functions, as shown. Error bars are standard errors of the means. Data from previous comparable studies are also shown (as described in the text). A comparison of the data collected with the procedure usually followed in this laboratory (constant force with one presentation, open circles) and that described most often in the literature (constant indentation with ad-lib presentations, filled squares) can be seen. 
ence absolute threshold when all other conditions are held equal (Craig \& Sherrick, 1969, p. 101). Nevertheless, the shape of the group loudness functions were not affected by the method that was used to maintain the skin-contactor relationship.

To examine the effect of the procedural variation in the number of stimulus presentations, the curves in the top, middle, and bottom portions of Figure 2 can be compared. The number of stimulus presentations appears to have had no effect on the functional relationship between stimulus intensity and perceived magnitude [force, $F(2,338)=$ $0.592, p>.05$; indentation, $F(2,323)=0.461, p>$
$.05]$. There is, however, a small but statistically significant difference among the overall levels of estimates for the constant-force condition, with the one-presentation stimuli judged as less intense than the others [one vs. three presentations, $F(1,227)=7.78, p \leq .05$; one presentation vs. ad lib, $F(1,227)=8.12, p \leq .05$ ]. There was no such difference found in the constant-indentation data $[F(2,325)=0.102, p>.05]$. It is unclear why the stimuli presented three or more times would necessarily feel louder. One process, enhancement, does involve an increase in perceived intensity with repeated stimuli, but enhancement typically does not take place unless stimuli

\section{Constant Force - Lower Limb Slope/Upper Limb Slope}

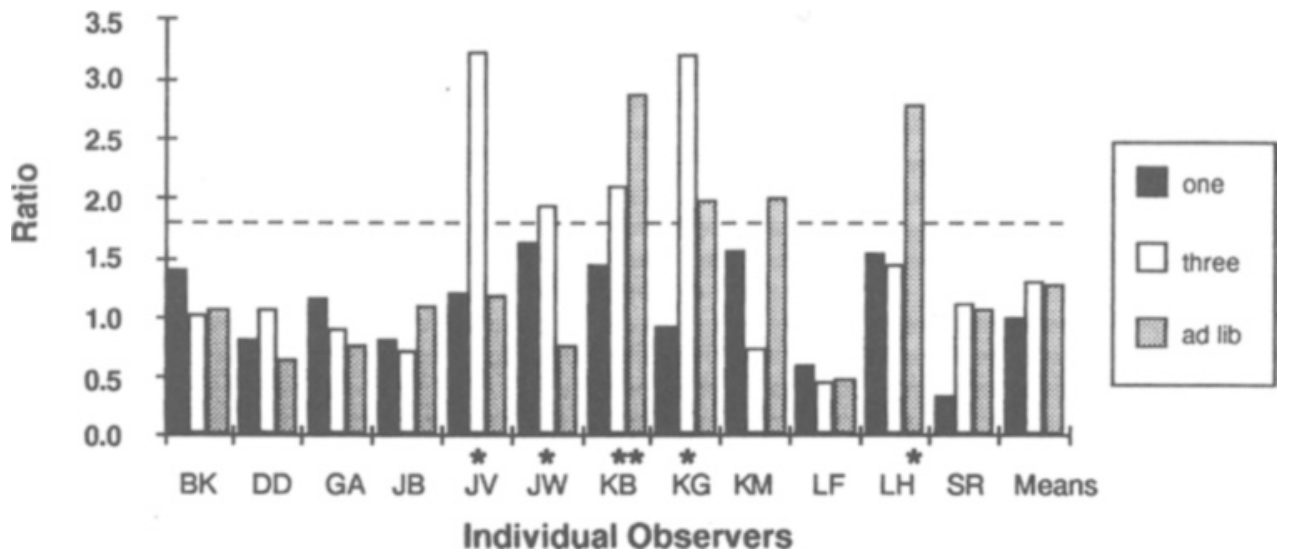

\section{Constant Indentation - Lower Limb Slope/Upper Limb Slope}

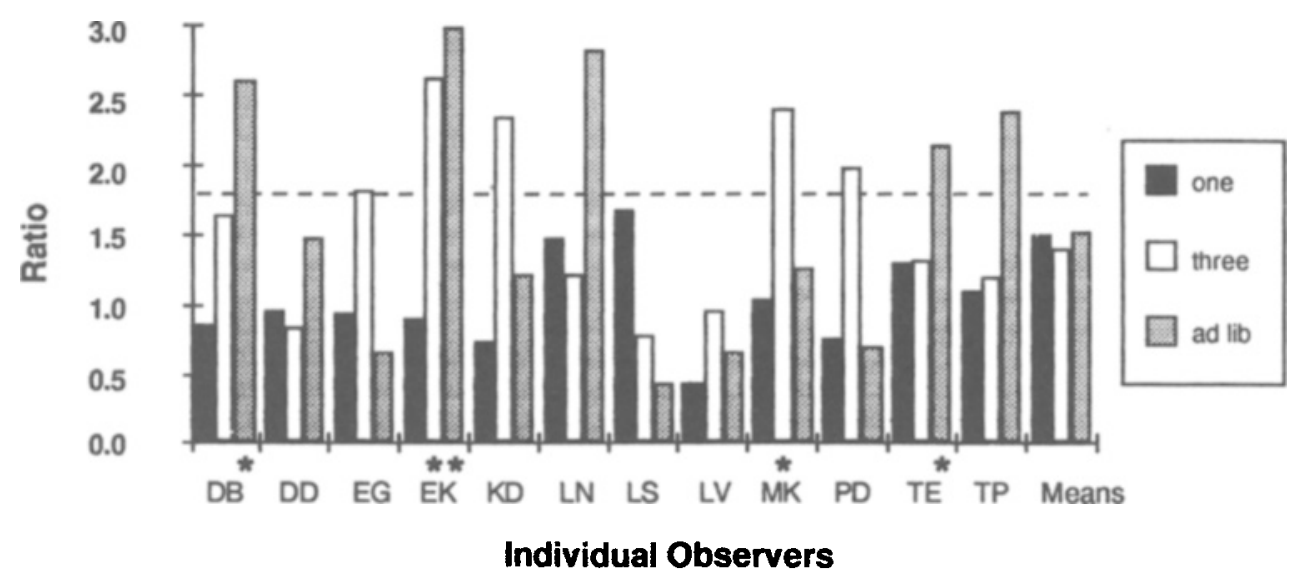

Figure 3. Histograms plotting the ratios of the lower:upper limb exponents for each individual and repetition condition for the experiment in which force was held constant (upper graph) or indentation was held constant (lower graph). One of the criteria for a two-limbed function, a ratio of 1.8 or greater, is indicated by the broken line. A few individual functions met this criterion-those that did were for conditions in which the stimuli were repeated more than once. Furthermore, of those, only the ratios marked with asterisks $\left(^{*}\right)$ were found to meet the statistical test as well. Note that none of the group means met these criteria. 
fall within 500 msec of one another (Verrillo \& Gescheider, 1975). The bursts in this study were presented with an interstimulus interval of $1,500 \mathrm{msec}$. As was the case with the other manipulated variable of coupling arrangement, repetition condition does not appear to have had an effect on the shape of the group loudness functions.

Because it is possible that averaging the data could have minimized or distorted changes in slope (Van Doren, 1990; Zwislocki, 1983), the roles of the number of stimulus presentations and coupling arrangement on the shapes of the loudness functions were also analyzed by examining the data from the individual observers. The appropriate descriptions of the shapes of the loudness functions (one- or two-limbed) were determined by applying the two criteria described previously. This analysis revealed different patterns of results for the single- and multiplepresentation conditions (three and ad lib; see Figure 3). The data from only 1 observer in the one-presentation condition met the statistical criterion for a two-limbed function, whereas none of the cases produced a 1.8:1 ratio between the lower- and upper-limb slopes. In the multiplepresentation data, there were scattered instances in which one or the other criterion was met. Both slope and statistical criteria, however, were met in only 6 of the 36 constant-force cases and 5 of the 36 constant-indentation cases (as indicated by the asterisks in Figure 3). These were distributed across observers; some of the individuals yielded two-limbed functions in one multiplepresentation condition and not in the other. ${ }^{6}$ Note that only about $23 \%$ of the cases in the multiple-presentation conditions showed this tendency. These data suggest that the shapes of the sinusoidal loudness functions are primarily affected by the number of stimulus presentations within a trial, but not to the degree that would account for the difference between the data from Verrillo (1974) shown in Figure 1 and those in the upper panel of Figure 2.

\section{DISCUSSION}

We have found that one procedural factor, number of repetitions, does appear to influence the shapes of the magnitude estimation functions in the lower intensity region for some individuals. It is unclear what the shared characteristics of those observers might be to produce this effect. The second procedural factor, the skin-contactor coupling condition, did not appear to affect the shapes of the functions, although the absolute level (loudness) was consistently greater for constant indentation, probably owing to a greater net force. What has been shown is that neither obvious instructional nor procedural differences seem to consistently influence the form of the function in the low-intensity region in which small changes can have such dramatic implications for the loudness of these stimuli.

There are a number of ways in which a steep lower limb could be generated intentionally: through adaptation (Gescheider \& Wright, 1968), introduction of noise (Sherrick, 1960; S. S. Stevens, 1966; Zwislocki, 1965), and recruitment (Hellman \& Hellman, 1975; S. S. Stevens \& Guirao, 1967; Verrillo, 1974). We are unaware, however, of any manipulations (other than mathematical) that would flatten the rate of growth of a loudness function to account for the functions we have obtained. So the question still remains as to why the present data do not show a steep lower range, especially when using the procedure of constant force with one presentation of the stimulus, but also when using the more traditional methodologies. A number of possible explanations have been entertained and rejected. One might be that the measured thresholds were higher than true threshold. If this were the case, the series could have been run in a range of intensities that allowed examination of the upper limb only. However, it was the case that the measured thresholds were somewhat lower than normally reported. Another explanation that has been advanced was that our observers experienced "fraction fear," which is a reluctance to generate magnitude estimates smaller than a value of 1.0. This situation occurs, for example, in young children who are not familiar with numeric values below unity (see, e.g., Collins \& Gescheider, 1989, Figure 2; Zwislocki \& Goodman, 1980, Figure 12). Examination of Figure 2 in the present paper, however, illustrates that the observers routinely produced estimates smaller than 1 in response to stimuli in the 5-20 dB SL range. It is also unlikely that the absence of two-limbed functions found here can be attributed to procedural bias, because the majority of such biases produce steepening in loudness curves or affect the entire intensity range and do not differentially affect the lower intensity range (Poulton, 1968, 1979).

Our pattern of results does not fall easily into any of the models that attempt to describe the shape of the lower region of the loudness function. Most of these models propose physiological processes for the change in rate of loudness growth shown in Figure 1 (Békésy, 1955; Gescheider \& Wright, 1968; Marks \& J. C. Stevens, 1968; S. S. Stevens, 1959, 1966; Verrillo, 1974; Zwislocki, 1965, 1974), whereas others suggest that numerical response bias plays a role (Dawson \& Miller, 1978; Poulton, 1968). The fact that there were differences in the shapes of the functions between and within the data of the individual observers conflicts with physiological models that are based upon properties that should hold true for every individual and each condition. Models suggesting that the steep lower limb is a by-product of number usage leave more room for the possibility that individuals may differ from time to time, from one another, and from study to study. The implication is that, at least for the vibrotactile stimuli studied here, it is a higher cognitive process that produces steep lower limbs in growth of magnitude functions, not receptor characteristics and mechanics.

\section{REFERENCES}

BÉKÉSY, G., VON (1955). Human skin perception of traveling waves similar to those on the cochlea. Journal of the Acoustical Society of America, 27, 830-841. 
Bernstein, L. E., Schechter, M. B., \& Goldstein, M. H., JR. (1986). Child and adult vibrotactile thresholds for sinusoidal and pulsatile stimuli. Journal of the Acoustical Society of America, 80, 118-123.

Cholewiak, R. W., Sherrick, C. E., \& Collins, A. A. (1992). Princeton cutaneous research project (Tech. Rep. No. 59). Princeton: Princeton University, Department of Psychology.

Collins, A. A., \& Cholewiak, R. W. (1989a). A comparison between the growths of loudness of vibrotactile pulse and sinusoidal stimuli. Journal of the Acoustical Society of America, 85(Suppl. 1), S64.

Collins, A. A., \& Cholewiak, R. W. (1989b). Regression effects in magnitude judgments of line length, and vibrotactile sine and pulse loudness. Journal of the Acoustical Society of America, 86(Suppl. 1), S96.

Collins, A. A., \& Gescheider, G. A. (1989). The measurement of loudness in individual children and adults by absolute magnitude estimation and cross-modality matching. Journal of the Acoustical Society of America, 85, 2012-2021.

Craig, J. C., \& Sherrick, C. E. (1969). The role of skin coupling in the determination of vibrotactile spatial summation. Perception \& Psychophysics, 6, 97-101.

Dawson, W. E., \& Miller, M. E. (1978). Inverse attribute functions and the proposed modifications of the power law. Perception \& Psychophysics, 24, 457-465.

GESCHEIDER, G. A. (1976). Evidence in support of the duplex theory of mechanoreception. Sensory Processes, 1, 68-76.

GescheIDER, G. A., \& WRiGHT, J. H. (1968). Effects of sensory adaptation on the form of the psychophysical magnitude function for cutaneous vibration. Journal of Experimental Psychology, 77, 308-313.

Green, B. G., \& CraIG, J. C. (1974). The roles of vibration amplitude and static force in vibrotactile spatial summation. Perception \& Psychophysics, 16, 503-507.

Hellman, W. S., \& Hellman, R. P. (1975). Relation of the loudness function to the intensity characteristics of the ear. Journal of the Acoustical Society of America, 57, 188-192.

LAMORÉ, P. J. J., \& KEEMinK, C. J. (1988). Evidence for different types of mechanoreceptors from measurements of the psychophysical threshold for vibrations under different stimulation conditions. Journal of the Acoustical Society of America, 83, 2339-2351.

LAVERTY, M. (1981). The growth of vibrotactile loudness at several body sites: Effects on the perception of two successive stimuli. Unpublished senior thesis, Princeton University, Princeton, NJ.

MARKS, L. E. (1979). Summation of vibrotactile intensity: An analog to auditory critical bands? Sensory Processes, 3, 188-203.

Marks, L. E., \& Stevens, J. C. (1968). The form of the psychophysical function near threshold. Perception \& Psychophysics, 4, 315-318.

Poulton, E. C. (1968). The new psychophysics: Six models for magnitude estimation. Psychological Bulletin, 69, 1-19.

Poulton, E. C. (1979). Models for biases in judging sensory magnitude. Psychological Bulletin, 86, 777-803.

SHERRICK, C. E. (1960). Observations relating to some common psychophysical functions as applied to the skin. In G. R. Hawkes (Ed.), Symposium on Cutaneous Sensitivity (Rep. No. 424, pp. 147-158). Fort Knox, TN: U.S. Army Medical Research Laboratory.

Stevens, S. S. (1959). Tactile vibration: Dynamics of sensory intensity. Journal of Experimental Psychology, 57, 210-218.

STEVENS, S. S. (1966). Power group transformations under glare, masking, and recruitment. Journal of the Acoustical Society of America, 39, $725-735$

Stevens, S. S. (1968). Tactile vibration: Change of exponent with frequency. Perception \& Psychophysics, 3, 223-228.

Stevens, S. S. (1975). Psychophysics: Introduction to its perceptual, neural, and social prospects. New York: Wiley.

Stevens, S. S., \& Guirao, M. (1967). Loudness functions under inhibition. Perception \& Psychophysics, 2, 459-465.

VAN DoREN, C. L. (1990). The effects of a surround on vibrotactile thresholds: Evidence for spatial and temporal independence in the nonPacinian I (NP I) channel. Journal of the Acoustical Society of America, 87, 2655-2661.

VERRILLO, R. T. (1962). Investigation of some parameters of the cutaneous threshold for vibration. Journal of the Acoustical Society of America, 34, 1768-1773.
VERRILLO, R. T. (1966). Effect of spatial parameters on the vibrotactile threshold. Journal of Experimental Psychology, 71, 570-575.

VERRILIO, R. T. (1974). Vibrotactile intensity scaling at several body sites. In F. A. Geldard (Ed.), Cutaneous communication systems and devices (pp. 9-14). Austin, TX: The Psychonomic Society.

VERRILLO, R. T. (1982). Effects of aging on the suprathreshold responses to vibration. Perception \& Psychophysics, 32, 61-68

Verrillo, R. T., \& Capraro, A. J. (1975). Effect of stimulus frequency on subjective vibrotactile magnitude functions. Perception \& Psychophysics, 17, 91-96.

Verrillo, R. T., \& Chamberlain, S. C. (1972). The effect of neural density and contactor surround on vibrotactile sensation magnitude. Perception \& Psychophysics, 11, 117-120.

Verrillo, R. T., Fraioli, A. J., \& SMith, R. L. (1969). Sensation magnitude of vibrotactile stimuli. Perception \& Psychophysics, 6, 366-372.

VerRllo, R. T., \& Gescheider, G. A. (1975). Enhancement and summation in the perception of two successive vibrotactile stimuli. Perception \& Psychophysics, 18, 128-136.

Wiggans, G. N., ANDrews, J. S., \& SaHgal, A. (1983). COMPREG: A BASIC program to test for differences between several linear regression lines. Behavior Research Methods \& Instrumentation, 15, 609-610.

WoODWARD, R. H. (1972). Linear relationship between two variables. In O. L. Davies \& P. L. Goldsmith (Eds.), Statistical methods in research and production (pp. 178-236). New York: Hafner.

ZWISLOCKI, J. J. (1965). Analysis of some auditory characteristics. In R. D. Luce, R. R. Bush, \& E. Galanter (Eds.), Handbook of mathematical psychology (Vol. 3, pp. 1-97). New York: Wiley.

ZWISLOCKI, J. J. (1974). A power function for sensory receptors. In H. R. Moskowitz (Ed.), Sensation and measurement (pp. 185-197). Dordrecht, Holland: D. Reidel.

ZWISLOCKI, J. J. (1983). Group and individual relations between sensation magnitudes and their numerical estimates. Perception \& Psychophysics, 33, 460-468.

ZWISLOCKI, J. J., \& Goodman, D. A. (1980). Absolute scaling of sensory magnitudes: A validation. Perception \& Psychophysics, 28, 28-38.

\section{NOTES}

1. Throughout this paper, exponents of power functions (slopes of the curves) will be computed in terms of amplitude of displacement (i.e., $\mathrm{dB}=20 \cdot \log$ [amplitude/reference]). It should be noted that since 1972, Verrillo and his associates have reported slopes of subjective magnitude curves calculated in terms of intensity (i.e., $\mathrm{dB}=10 \cdot \log$ [amplitude/reference]; Verrillo \& Chamberlain, 1972, p. 118). The consequence is that, in order to maintain consistency, the slopes reported here from studies by these authors are twice as large as those appearing in the original papers.

2. Threshold functions obtained with $20 \mathrm{~g}$ of constant force imposed on a 7-mm-diam contactor on the skin are usually found to be equivalent to those produced by a stationary 7 -mm-diam contactor with a 0.5 $\mathrm{mm}$ indentation on the weighted hand (Cholewiak, Sherrick, \& Collins, 1992; Lamoré \& Keemink, 1988). However, when parameters such as the area of the contactor are allowed to vary, differences appear in the threshold values, and there has been some debate about which condition produces the more accurate representation of the response of the skin (Craig \& Sherrick, 1969; Green \& Craig, 1974; Lamoré \& Keemink, 1988)

3. It is possible that with this arrangement, the actual indentation of the contactor into the skin could be something less than $0.5 \mathrm{~mm}$ for some observers. This might allow for loss of contact between the skin and contactor at high-intensity levels (Verrillo, 1966). As a result, the full displacement of the high-intensity stimuli might not be felt, resulting in smaller estimates of their loudness and a flattening of the upper portion of the loudness curve. If this situation occurred, its effect would be to exaggerate any differences in the slopes of the lower and upper portions of the loudness curve.

4. The curves were analyzed with the COMPREG BASIC program developed by Wiggans et al. (1983). COMPREG is comprised of a set of statistical tests taken from Woodward (1972) that assess the differ- 
ences among a number of regression lines on the basis of combined analyses of variance and covariance (regression) techniques. COMPREG was used for all comparisons between regression lines in the present paper.

5. All conditions failed the ratio test. However, the statistical criterion for two limbs was met by the three-presentation and ad-lib conditions of both constant force and constant indentation [three-presentation, force, $F(1,8)=8.27, p<.05$; three-presentation, indentation, $F(1,8)=$ $6.57, p<.05$; ad lib, force, $F(1,8)=7.03, p<.05$; ad lib, indentation, $F(1,7)=6.94, p<.05]$.

6 . The possibility that production of two-limbed functions was a function of experience within the session regardless of repetition condition was examined. In the constant-force study, two-limbed functions were found in $25 \%$ of the first-block data, $8 \%$ of the second, and $17 \%$ of the third. In the constant-indentation study, two-limbed functions were found in $25 \%$ of the first-block data, $17 \%$ of the second, and $0 \%$ of the third.

7. Verrillo's (1974) data show a lower:upper exponent ratio of 2.08 and meet both two-limb criteria. The slopes of the best-fit functions for these two limbs are significantly different $[F(1,5)=264.71, p<.05]$.

(Manuscript received October 2, 1992; revision accepted for publication August 11, 1993.) 